Löcher bekommen haben «. Die Justiz habe aber auch keine andere Wahl gehabt: "Entweder stellt die Justiz die Illegalität der Bullen fest, oder sie begibt sich selbst in die Illegalität. «

Die Öffentlichkeit hat den Unterschied bemerkt. Nicht nur, daß das »Bombenurteil « (Frankfurter Rundschau) bei den Presseberichterstattern selber wie eine Bombe einschlug, nicht nur daß selbst Polizisten im Gerichtssaal zusammenzuckten, daß die Richter beim letzten Satz mit fliegendem Start und wehenden Uniformen ins Beratungszimmer flüchteten und die Staatsanwaltschaft das Urteil "schädlich" fand: Eine Flut des Protests erreichte die Verteidiger, an Werner Hoppe selber schickte u. a. die Belegschaft einer Kölner Firma Solidaritätsbekundungen. Die neue Qualität des Urteils nicht erkannt hat jedoch diejenige Presse, "die bei einer Kritik stehenbleibt, die nicht die Willkür und den Terror sieht, nicht die gesamte Faschisierung, sondern die nur den Fleck auf dem Rechtsstaat< kritisiert und sich nur ausläßt: >6 Jahre wären doch genug gewesen<.

Peggy Parrass

\title{
Zentrales Informationsbüro (ZIB)
}

Im März dieses Jahres haben sich unter dem Namen ২Zentrales Informationsbüror Rechtsanwälte, die insbesondere politische Strafverteidigungen übernehmen und Juristen zusanmengeschlossen, um ihre Arbeit durch gegenseitige Information zu erleichtern und zu koordinieren.

Zunächst wurde ein umfangreicher Katalog der Rechtsbereiche aufgestellt, die für diese Anwaltspraxen besonders relevant sind, nämlich u. a. Straf- und Strafprozeßrecht, Verfassungs- und Verwaltungsrecht, Schul- und Hochschulrecht, Kriegsdienstverweigerungs- und Ersatzdienstrecht, Ausländerrecht, Mietrecht, Arbeitsrecht, Sozialrecht, Versammlungsrecht.

Inzwischen hat die Sammlung des jeweiligen einschlägigen Materials (Entscheidungen, Aufsätze, etc.), das von allen Beteiligten und der Zentralstelle in Heidelberg gesammelt und von letzterer aufgearbeitet wird, begonnen.

Die Auswertung und Weitergabe dieses Materials erfolgt kontinuierlich an alle Mitglieder des ZIB. Zusätzlich kann unter Angabe des gesuchten Problembereiches weiteres Material vom ZIB oder mit dessen Hilfe von einzelnen Mitgliedern angefordert werden.

Barbara Dietrich

\section{Wirtschaftskriminalität - am Beispiel der Kundenkreditbank}

I.

Aus den Kreditbedingungen für Abzahlungsgeschäfte der Kundenkreditbank KGaA., Düsseldorf (Fassung vom I. 8. 197I):

7. Der Restsaldo ist zur sofortigen Rückzahlung fällig, wenn die Kreditnehmer mit einer Rate länger als 20 Tage in Verzug geraten.

17. Gerichtsstand für alle Streitigkeiten der Bank gegen Kreditnehmer, Mitverpflichtete, Bürgen oder Garantiegeber ist der Sitz der Niederlassung der KKB ... 
Im Falle der Abtretung an die TZ-Inkasso-Treuhandgesellschaft FIDUCIA W. Pescher, Mannheim, ist Gerichrsstand Mannheim.

II.

Aus dem Abzahlungsgesetz vom I6. Mai I 894 (Fassung vom I. 9. 1969):

$\$ 4$ Abs. 2: Die Abrede, daß die Nichterfüllung der dem Käufer obliegenden Verpflichtungen die Fälligkeit der Restschuld zur Folge haben solle, kann rechtsgültig nur für den Fall getroffen werden, daß der Käufer mit mindestens zwei aufeinanderfolgenden Teilzahlungen ganz oder teilweise in Verzug ist und der Betrag, mit dessen Zahlung er im Verzug ist, mindestens dem zehnten Teile des Kaufpreises der übergebenen Sache gleichkommt.

$\$ 6 a$ Abs. r: Für Klagen aus Abzahlungsgeschäften ist das Gericht ausschließlich zuständig, in dessen Bezirk der Käufer zur Zeit der Klageerhebung seinen Wohnsitz, in Ermangelung eines solchen seinen gewöhnlichen Aufenthaltsort hat.

III.

An die

Staatsanwaltschaft

beim Landgericht Gießen

Hiermit erstatte ich

Strafanzeige gegen Unbekannt

wegen Nötigung.

Die Kundenkreditbank KGaA, Düsseldorf (KKB) - laut FAZ vom 14. 2. 1972 größte deutsche Teilzahlungsbank - verwendet bei Abzahlungsgeschäften die "KKB-Bedingungen vom I. August 197I ", die ich in der Anlage als Photokopie beifüge.

Bei der Durcharbeitung dieser Bedingungen habe ich gefunden, daß wenigstens zwei der darin enthaltenen Klauseln rechtswidrig sind. Nummer 7 der Bedingungen verstößt gegen die zwingende Schutzvorschrift des $\$ 4$ Abs. $2 \mathrm{AbzG}$, die eine Verfallklausel nur für den Fall des Verzuges »mit mindestens zwei aufeinanderfolgenden Teilzahlungen « zuläßt. Nummer 17 verstößt gegen die - durch Gesetz vom I. 9. 1969 (BGBl. I S. I 54 I) eingeführte - gleichfalls zwingende Schutzvorschrift des $\$ 6 \mathrm{a} A \mathrm{bzG}$, wonach ausschließlicher Gerichtsstand der Wohnort des Käufers ist.

$\mathrm{Daß}$ diese Klauseln vor Gericht für nichtig erklärt werden müßten, steht für mich fest. Andererseits nehme ich mit Sicherheit an, daß die KKB diese Klauseln regelmäßig im Umgang mit Kunden anwendet. Das bedeutet, daß die KKB androht, beim Verzug um 20 Tage werde der Gesamtbetrag fällig und im Streitfalle werde das vom Kunden möglicherweise weit entfernte Gericht zuständig sein. Wenigstens die rechtsunkundigen Kunden - die die Nichtigkeit der Klauseln nicht kennen - werden sich durch die Drohung der KKB einschüchtern lassen und sich im Regelfall ohne Anrufung eines Gerichts den Forderungen der KKB beugen.

Damit ist m. E. der objektive Tatbestand einer Nötigung, wenn nicht gar Erpressung gegeben. Auch wenn die Kunden materiellrechtlich möglicherweise im Unrecht sind, darf die KKB nicht rechtswidrige Mittel einsetzen und die Rechtsunkenntnis der Kunden ausnutzen, um ihre Interessen durchzusetzen. Ein vertretbares Zweck-Mittel-Verhältnis scheitert schon an der Rechtswidrigkeit des Mittels. 
Was den subjektiven Nötigungstatbestand angeht, ist folgendes zu sagen. $\ 4$ AbzG gilt in der heutigen Fassung schon seit Jahren, $\$$ 6a seit I 969. Die KKB-Bedingungen liegen in der Fassung von 1971 vor. Sie sind - wie die Durcharbeitung zeigt - unter genauester Kenntnis abzahlungsrechtlicher Zusammenhänge, sicher von erfahrenen Juristen, abgefaßt. Daß diesen die Vorschriften des AbzG, insbesondere die Anderung von 1969 , entgangen sein sollte, ist vollkommen ausgeschlossen. Die für die Bedingungen Verantwortlichen wissen, daß die Klauseln rechtswidrig sind. Sie wissen auch, daß jedes Gericht sie - wenn es angerufen wird - für nichtig erklärt. Die Verantwortlichen wissen daher auch, daß die unwirksamen Bedingungen überhaupt nur dann einen Sinn haben, wenn sich Kunden ihnen aus Rechtsunkenntnis beugen und daher nicht ein Gericht anrufen. Damit sind auch die subjektiven Voraussetzungen einer Nötigung gegeben.

Der Einwand, die Kunden könnten sich ja gegen die rechtswidrigen Klauseln zur Wehr setzen, schließt eine Nötigung nicht aus. Denn gerade für den Fall, daß die Kunden diese Unwirksamkeit nicht kennen, sind die Bedingungen geschaffen. Gerade die Rechtsunkenntnis des Publikums wird also ausgenutzt, um wirtschaftlichen Druck auszuüben.

Allein die vorgetragenen Umstände bieten m. E. zureichende tatsächliche Anhaltspunkte für strafbare Handlungen, die die Staatsanwaltschaft nach $\$$ I 52 Abs. 2 $\mathrm{StPO}$ zum Einschreiten verpflichtet. Es dürfte nicht schwerfallen festzustellen, in welchen Fällen die beanstandeten Klauseln angewandt worden sind, ohne $\mathrm{daB}$ sich die Betroffenen dagegen gerichtlich zur Wehr gesetzt haben. In diesen Fällen läge vollendete Nötigung vor. Auch der Täterkreis wird sich ohne Schwierigkeiten ermitteln lassen. Es wird sich dabei um kaufmännisch Verantwortliche der KKB und deren Rechtsstab handeln.

Ich glaube, daß es ungewöhnlich ist, Fälle wie den hier angezeigten zu verfolgen. Ein wirksamer Verbraucherschutz auch durch die Strafverfolgungsbehörden muß aber gerade dort einsetzen, wo der Gesetzgeber zwar zum Schutz der Verbraucher tätig geworden ist, wo aber diese Schutzvorschriften von den wirtschaftichen Interessenten durch gezielte Ausnutzung der Rechtsunkenntnis der Kunden umgangen und damit ihrer faktischen Wirkung beraubt werden.

Ich bitte Sie, mich über den weiteren Gang des Verfahrens auf dem laufenden zu halten. Insbesondere weise ich auf $\$$ I 7 I S. I StPO hin.

(U. Mückenberger)

IV.

Staatsanwaltschaft Düsseldorf

Geschäfts-Nr.: I I Js $929 / 72$

${ }_{4}$ Düsseldorf, den 27.6. I 972

Herrn

Gerichtsreferendar

Ulrich Mückenberger

63 Gießen

Roonstr. I6

Betr.: Ermittlungsverfahren gegen Dr. Walter Kaminski

als Vorstandsvorsitzender der Kundenkreditbank in Düsseldorf

wegen Verdachts der Nötigung

Bezug: Ihre an die Staatsanwaltschaft Gießen gerichtete Strafanzeige vom 10. 3. 1972 
Sehr geehrter Herr Mückenberger,

der von Ihnen angezeigte Sachverhalt reicht nicht hin, den Tatbestand des $\subseteq$ $240 \mathrm{StGB} z u$ begründen.

$\mathrm{Z}$ war mag es zutreffen, daß die Ziffern 7 und 17 der KKB-Kredit-Bedingungen gegen die $\$ \Phi_{4} \mathrm{Abs} .2$ und $6 \mathrm{a} \mathrm{AbzG}$ verstoßen.

Diese Klauseln sind jedoch weder sittlich so mißbilligenswert, daß sie ein gesteigertes, als Vergehen strafwürdiges Unrecht begründen könnten, noch sind sie als sozialwidrig anzusehen.

Eine strafrechtliche verfolgbare Nötigung ist mithin nicht gegeben

Deshalb muß das Verfahren eingestellt werden.

Hochachtungsvoll

Grasnick

Erster Staatsanwalt

\section{$V$.}

Mit der Anzeige wurde die Bereitschaft der staatlichen Instanz getestet, zwingende Konsumentenschutzbestimmungen mit den ihr zur Verfügung stehenden Mirteln durchzusetzen. Dem geht die früher formulierte These voraus, daß die rechtsförmige Ausweitung des Konsumentenschutzes keineswegs mit deren faktischer Durchsetzung gleichzusetzen ist, wie die Rechtsillusion glauben macht (vgl. Mückenberger und Hart KJ 3/71, S. 242, 246, 277). Der Test auf die Ernsthaftigkeit des Konsumentenschutzes liegt deshalb in der Prüfung der Sanktionen, denen durch Gesetzgebung und Justiz illegalisiertes Profitstreben ausgesetzt ist. Daß bei den KKB-Kreditbedingungen ein Fall von Wirtschaftskriminalität vorliegt, dürfte sich aus dem Text der Anzeige eindeutig ergeben. Mit ihrer Einstellungsverfügung verweigert die Staatsanwaltschaft die Sanktionierung illegalisierten Verhaltens und damit den Konsumentenschutz.

Die Staatsanwaltschaft läßt den dem Gesetzgeber abgerungenen Konsumentenschutz durch Sittlichkeits- und Sozialerwägungen leerlaufen, was im Ergebnis bedeutet, daß sie sich zum Zensor des Gesetzgebers macht. Sie lehnt die Verfolgung eines durch Gesetz eindeutig mißbilligten Verhaltens ab, weil es sittlich nicht genügend mißbilligenswert sei, und spricht dem Verstoß gegen eine gesetzgeberische Sozialmaßnahme die Sozialwidrigkeit ab.

Kriminelles Verhalten wie das der KKB hat einen enormen Multiplikator-Effekt, weil die Bedingungen der größten deutschen Teilzahlungsbank millionenfach angewandt werden. Dergleichen als nicht sozialwidrig bezeichnen kann nur, wer sich voll mit den Kapitalinteressen identifiziert. Wenn man die Großzügigkeit der Staatsanwaltschaft gegenüber der KKB einmal vergleicht mit der Großzügigkeit, das Verhalten leichtfertiger Ratenkäufer als »Eingehungsbetrug « ( $=$ Vortäuschung der Zahlungsfähigkeiten als Betrugshandlung) einzustufen, und mit der Kleinlichkeit, das Verfahren gegen kleine Warenhausdiebe einzustellen, und wenn man über die unterschiedliche Behandlung nachdenkt, ist man dem Problem der Klassenjustiz auf der Spur.

Bewußt parteiisches Verhalten der handelnden Personen wird dabei keine allzu große Rolle spielen. Eine Anzeige der vorliegenden Art stellt einen Staatsanwalt schon aufgrund seiner normalen Arbeitsbedingungen vor praktisch kaum lösbare Schwierigkeiten. Es müßte die Kripo auf Herren ansetzen, die ihm (und erst recht den Polizisten) im Regelfall an Geld, Einfluß, Ansehen und Gewandtheit weit überlegen sind und denen selten etwas wird nachgewiesen werden können. 
Schon die Schwierigkeit, einen "Schuldigen " zu ermitteln, ist viel größer als bei der Kleine-Leute-Kriminalität. Wen sollte man verantwortlich machen bei der weitverzweigten Organisation eines Großberiebes: den Rechtsstab, der doch nur als Werkzeug praktisches Wissen verfügbar macht, aber im wörtlichen Sinne verantwortungslos handelt - die Geschäftsführung, die von alledem nichts wissen will?

Dazu kommt die Schwierigkeit, Geschädigte ausfindig zu machen. Trotz des Offizialprinzips ist die Strafverfolgung im Regelfall nicht weniger auf die Gegenwehr des Betroffenen angewiesen als die zivile Rechtsverfolgung. Wo kein Kläger ist, ist kein Richter.

Das Strafrecht steht im Bann des individualistischen Schuldprinzips wie das Zivilrecht in dem der Privatautonomie. Dieses Prinzip selegiert das justizrelevante Verhalten. Kollektivität entzieht sich dem Prinzip. Das Problem ist nicht neu und nicht nur eines der Wirtschaftskriminalität, es tauchte auch z. B. bei den NS-Gewaltverbrechen und bei der Verfolgung von Demonstranten auf. Aber während bei den NS-Prozessen dem starken Offentlichkeitsdruck schlecht und recht Genüge getan wurde, indem kollektiver Terror in den Raster individuellen Unrechts gepreßt wurde, und während in den Studentenprozessen Generalabschreckung durch drakonische Einzelbestrafung geübt wurde, führt das Schuldprinzip im Falle der organisierten Wirtschaftskriminalität zur Abschirmung der Kapitalinteressen gegen öffentlichen Zugriff.

Vor diesem Hintergrund kann das Ergebnis des hier angestellten Tests nicht überraschen. Vor die Wahl gestellt, sich unabsehbaren Schwierigkeiten mit ungewissem, vielleicht blamablem Ausgang auszusetzen oder die Sache ad acta zu legen, entscheidet sich ein mit Routinetätigkeit stark belasteter Staatsanwalt für den letzteren Weg. Zur Erklärung dieses Ergebnisses braucht man gar nicht den professionellen Konservatismus eines Staatsanwalts zu bemühen. Schon die Bedingungen, unter denen er arbeitet, strukturieren seine Entscheidung im Sinne der vorherrschenden Interessen vor. Der Konservatismus liefert dafür nur noch die legitimatorische Formel.

Das Ergebnis des Tests ist, daß die Klauseln der KKB-Bedingungen, die sich offen über zwingende Konsumentenschutzbestimmungen hinwegsetzen, unangefochten weiterverwandt werden können. Der Strafrechtsweg ist mit der Einstellungsverfügung abgeschnitten. Eine Überprüfung dieser Verfügung kann ein Außenstehender nicht erzwingen. Jeder betroffene Abzahlungskäufer kann sich zwar im Zivilrechtsweg die Nichtigkeit der Klauseln bescheinigen lassen, wenn sie gegen ihn angewandt werden. Aber unter denen, gegen die die Klauseln angewandt werden, wird baum einer zu dieser rechtlichen Beurteilung in der Lage sein. Auch wenn sich jemand gegen die Klauseln zur Wehr setzt, hat das Zivilurteil nur Geltung für die Prozeßparteien, d. h. für die unzähligen übrigen Fälle bleiben die Klauseln in Geltung. Diese praktische Demonstration der Hilflosigkeit gegenüber offen rechtswidrigen Bedingungen straft Illusionen in Sachen Konsumentenschutz Lügen.

Ulrich Mückenberger 